\title{
EDITORIAL
}

\section{Special Issue: Photo- and Electro-Functional Polymers and Molecular Assemblies}

Polymer Journal (2017) 49, 1; doi:10.1038/pj.2016.111

Since the discovery by Shirakawa, Heeger and MacDiarmid that $S_{\text {conjugated polymers can be turned into electrically conducting }}$ materials, ${ }^{1,2}$ in the 1970 s, the field of organic $\pi$-conjugated materials has grown rapidly. The latest decade has seen a tremendous growth in research exploring organic and polymer semiconductors for various practical applications. Materials science on molecular and polymer materials will undoubtedly continue to be a key ingredient for the development of organic electronics and diverse photonic and electronic applications.

Recently, Polymer Journal has published special issues focusing on attractive topics in polymer science. ${ }^{3-9}$ We present this special issue of Polymer Journal on the topic of 'Photo- and Electro-Functional Polymers and Molecular Assemblies'. The broad and growing interest in utilizing such functional molecular, polymer and liquid-crystalline materials as active elements in various devices, including organic photovoltaic cells, transistors and light-emitting diodes, is well represented in this special issue. We have collected 14 Original Articles, 4 Focus Reviews and 4 Reviews from outstanding researchers in Japan, Taiwan, Korea, USA, France, Australia and India. This collection of papers can provide the readers with an overview of the recent progress in this exciting field.

As editors, we would like to thank all the authors and referees for their contributions to this special issue. Finally, we believe that this special issue will provide a valuable reference and perspective for many researchers working in polymer science and related fields.
Takuma Yasuda ${ }^{1}$, Wen-Chang Chen $^{2}$ and Takashi Kato ${ }^{3}$ ${ }^{1}$ INAMORI Frontier Research Center (IFRC), Kyushu University, Fukuoka, Japan; ${ }^{2}$ Department of Chemical Engineering, Institute of Polymer Science and Engineering, National Taiwan University, Taipei, Taiwan and ${ }^{3}$ Department of Chemistry and Biotechnology, School of Engineering, The University of Tokyo, Tokyo, Japan E-mail: yasuda@ifrc.kyushu-u.ac.jp or chenwc@ntu.edu.twor kato@chiral.t.u-tokyo.ac.jp

1 Shirakawa, H., Louis, E. J., MacDiarmid, A. G., Chiang, C. K. \& Heeger, A. J. Synthesis of electrically conducting organic polymers: halogen derivatives of polyacetylene, $(\mathrm{CH})_{x}$. J. Chem. Soc. Chem. Commun. 578-580 (1977).

2 Shirakawa, H. \& Ikeda, S. Infrared spectra of poly(acetylene). Polym. J. 2, 231-244 (1971).

3 Kato, T., Aoshima, S. \& Kikuchi, H. Special issue: self-assembled materials. Polym. J. 44, 451 (2012).

4 Asakura, T. \& Asano, A. Special issue: NMR of polymers: recent advances and innovations. Polym. J. 44, 733 (2012).

5 Kanaya, T., Sakurai, K. \& Takahara, A. Special issue: application of quantum beams to polymer science and engineering. Polym. J. 45, 2 (2013).

6 Venanzi, M. \& Kimura, S. Special issue: peptide materials. Polym. J. 45, 467 (2013).

7 Serizawa, T. Special issue: biorelated polymers and materials. Polym. J. 46, 435 (2014).

8 Kato, T., Kikuchi, H. \& Imai, H. Special issue: fusion materials: creative development of materials and exploration of their function through molecular control. Polym. J. 47, 77 (2015).

9 Tanaka, K., Serizawa, T., Chen, W.-C., Char, K. \& Kato, T. Special issue: polymer surfaces, interfaces and thin film. Polym. J. 48, 323 (2016). 